Supplementary Information:

\title{
Molecular Dynamics Simulations Provide Insight into the Loading Efficiency of Pro-Resolving Lipid Mediators Resolvin D1 and D2 in Cell Membrane-Derived Nanovesicles
}

\author{
Jeevan B. GC, Christopher T. Szlenk, Jin Gao, Xinyue Dong, \\ Zhenjia Wang, and Senthil Natesan* \\ Department of Pharmaceutical Sciences, College of Pharmacy and Pharmaceutical Sciences, \\ Washington State University, Spokane, WA 99202, USA \\ *senthil.natesan@wsu.edu
}

Table S1. Physicochemical properties of resolvin D1 and resolvin D2

\begin{tabular}{|l|c|}
\hline \multicolumn{2}{|c|}{$\begin{array}{c}\text { Resolvin D1 and Resolvin D2 } \\
\text { Physicochemical Properties }\end{array}$} \\
\hline Molecular weight & $376.5 \mathrm{~g} / \mathrm{mol}$ \\
\hline Number of rotatable bonds & 14 \\
\hline Number of H-bond donors & 4 (3 for ionized species) \\
\hline Number of H-bond acceptors & 5 \\
\hline Clog $P$ & 2.56 \\
\hline $\log D_{5.4}$ & 1.67 \\
\hline $\log D_{7.4}$ & -0.22 \\
\hline Species fraction at $\mathrm{pH} 5.4$ & $23: 77$ (neutral:ionized) \\
\hline Species fraction at $\mathrm{pH} 7.4$ & $0: 100$ (neutral:ionized) \\
\hline TPSA & 97.99 \\
\hline
\end{tabular}


Table S2. Details of the individual simulation steps

\begin{tabular}{|c|c|c|c|c|}
\hline Step & Ensemble & $\begin{array}{c}\text { Time step } \\
(\mathbf{f s} / \mathbf{s t e p})\end{array}$ & $\begin{array}{c}\text { Force constant } \\
(\text { Kcal/mol) }\end{array}$ & Simulation time \\
\hline 6.1 & NVT & 1 & 5.0 & $25 \mathrm{ps}$ \\
\hline 6.2 & NVT & 1 & 5.0 & $25 \mathrm{ps}$ \\
\hline 6.3 & NPT & 1 & 2.0 & $25 \mathrm{ps}$ \\
\hline 6.4 & NPT & 2 & 1.0 & $100 \mathrm{ps}$ \\
\hline 6.5 & NPT & 2 & 0.2 & $100 \mathrm{ps}$ \\
\hline 6.6 & NPT & 2 & 0.0 & $100 \mathrm{ps}$ \\
\hline Equilibration & NPT & 2 & 0.0 & 10 ns (each window) \\
\hline Umbrella Sampling & NPT & 2 & 1.5 & 50 ns (each window) \\
\hline
\end{tabular}
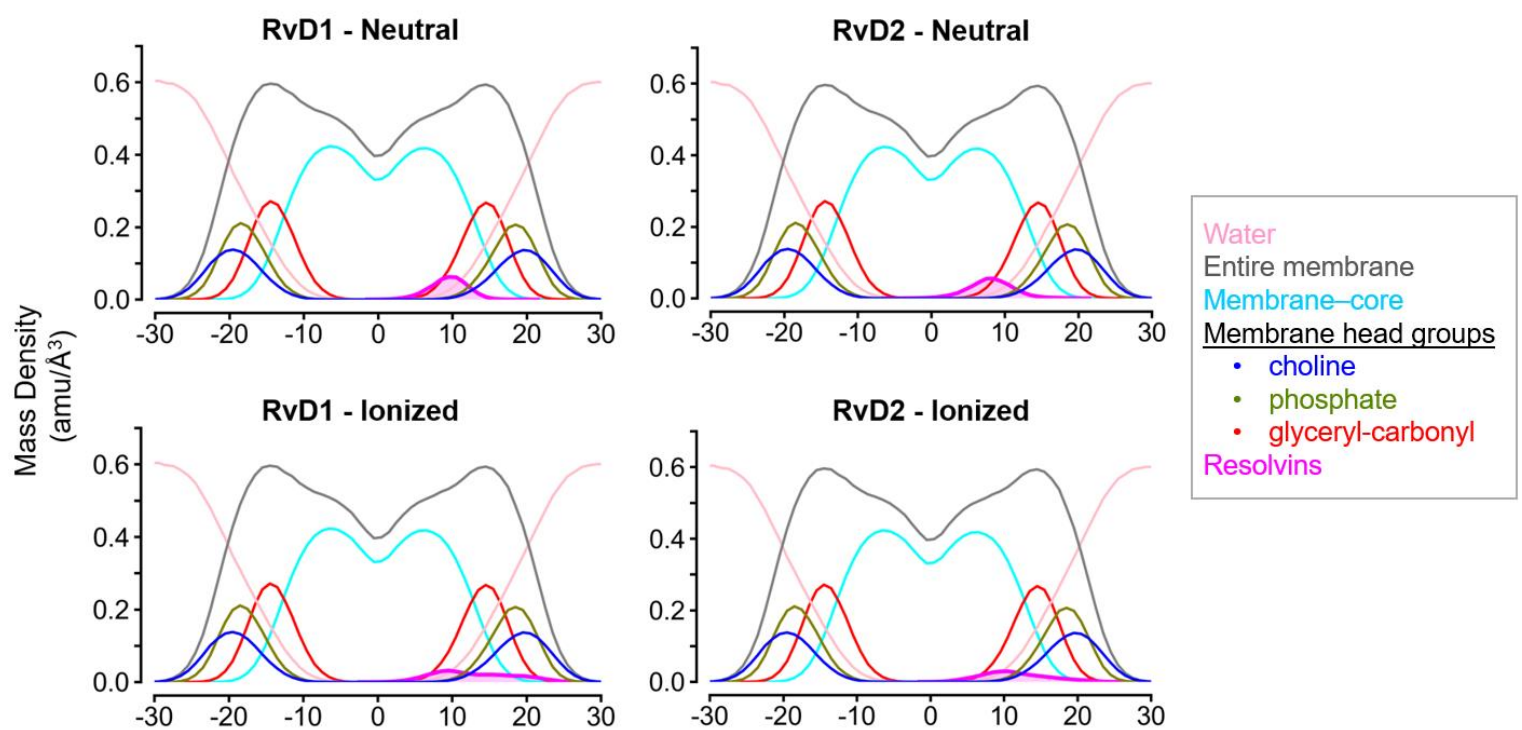

z - distance $(\AA)$

Fig. S1. Average mass density profiles of neutral and ionized species of RvD1 and RvD2 and the DMPC bilayer components along the entire bilayer normal (z-axis) showing water (light pink), entire membrane (dark gray), membrane core alkyl carbons (cyan), choline (blue), phosphates (olive green), glyceryl-carbonyls (red), and resolvins (magenta). The density profiles of resolvins were calculated for one bilayer leaflet and were symmetrized to the other side. The density values for resolvins were calculated at their respective $\left|z_{\min }\right| \pm 2 \AA$ and multiplied by 4 (arbitrary number) to provide visual clarity. The neutral species of both RvD1 and RvD2 have a narrow spread (indicating their parallel orientations to the bilayer plane) compared to the ionized species that have a broad spread (indicating their perpendicular orientations to the bilayer plane). 


\begin{tabular}{|c|c|c|c|c|c|}
\hline \multicolumn{6}{|c|}{ \%Occupancy } \\
\hline \multirow[b]{2}{*}{ ligand } & \multicolumn{2}{|c|}{ RvD1 } & \multicolumn{2}{|c|}{ RvD2 } & \multirow[b]{2}{*}{ 으ㅁㅡㅡ } \\
\hline & 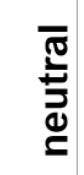 & 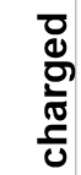 & 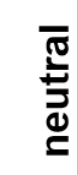 & $\begin{array}{l}\overline{0} \\
\frac{0}{0} \\
\frac{0}{0} \\
\frac{0}{0}\end{array}$ & \\
\hline \multirow[t]{4}{*}{$\mathrm{COOH}$} & 0.16 & 0.79 & 0.17 & 0.64 & C \\
\hline & 0.10 & 0.16 & 0.12 & 0.18 & $\mathrm{P}$ \\
\hline & 0.33 & 0.04 & 0.29 & 0.14 & G \\
\hline & 0.41 & 0.01 & 0.42 & 0.04 & A \\
\hline \multirow[t]{4}{*}{$\mathrm{OH}(1)$} & 0.26 & 0.28 & 0.30 & 0.32 & C \\
\hline & 0.18 & 0.17 & 0.12 & 0.15 & $\mathrm{P}$ \\
\hline & 0.29 & 0.32 & 0.32 & 0.29 & G \\
\hline & 0.27 & 0.22 & 0.26 & 0.24 & A \\
\hline \multirow[t]{4}{*}{$\mathrm{OH}(2)$} & 0.29 & 0.23 & 0.11 & 0.16 & C \\
\hline & 0.12 & 0.16 & 0.10 & 0.08 & $\mathrm{P}$ \\
\hline & 0.32 & 0.36 & 0.30 & 0.33 & G \\
\hline & 0.26 & 0.25 & 0.49 & 0.42 & A \\
\hline \multirow[t]{4}{*}{$\mathrm{OH}(3)$} & 0.25 & 0.31 & 0.25 & 0.18 & C \\
\hline & 0.10 & 0.10 & 0.09 & 0.04 & $\mathrm{P}$ \\
\hline & 0.35 & 0.33 & 0.29 & 0.36 & G \\
\hline & 0.29 & 0.26 & 0.36 & 0.42 & A \\
\hline
\end{tabular}

Fig S2. The percentage (\%) occupancy gives the fraction of simulation time (as a fraction of snapshots) during which the atom contacts were observed between the lipid groups and carboxyl and hydroxyl groups of the solutes. The functional groups were considered in contact if the distance between them was less than or equal to $4 \AA$. 
A

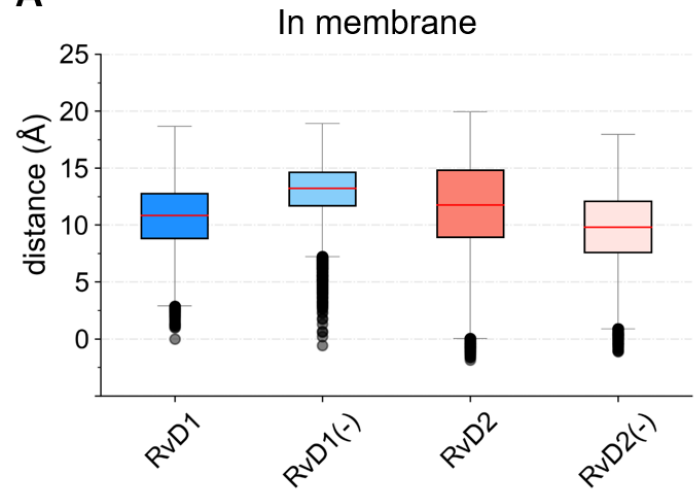

B

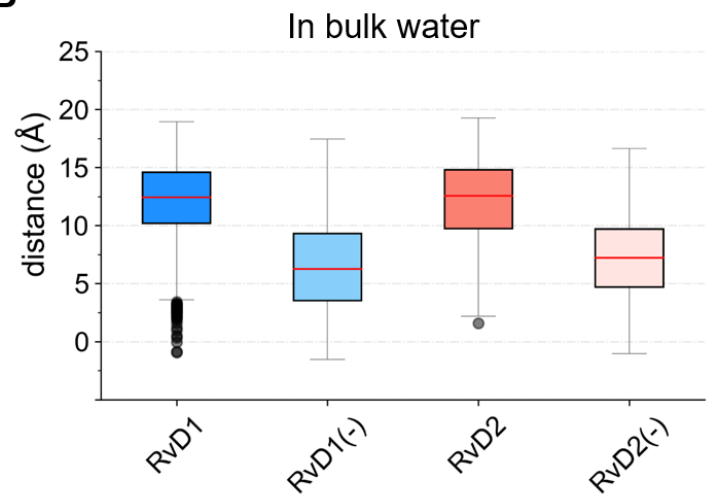

Fig S3. The end-to-end distance (in $\AA$ ) of the neutral and charged species of RvD1 and RvD2. This topological parameter was calculated by measuring the distance between the carboxyl group carbon atom and the carbon atom of the end methyl group and provide insights to the conformation of these lengthy molecules (either extended or bent conformations). Within the membrane, all the molecules seem to assume extended conformations. However, in bulk water, there is a significant difference between the neutral and charged species with neutral molecules assuming extended conformations while the charged molecules assumed bent conformations.

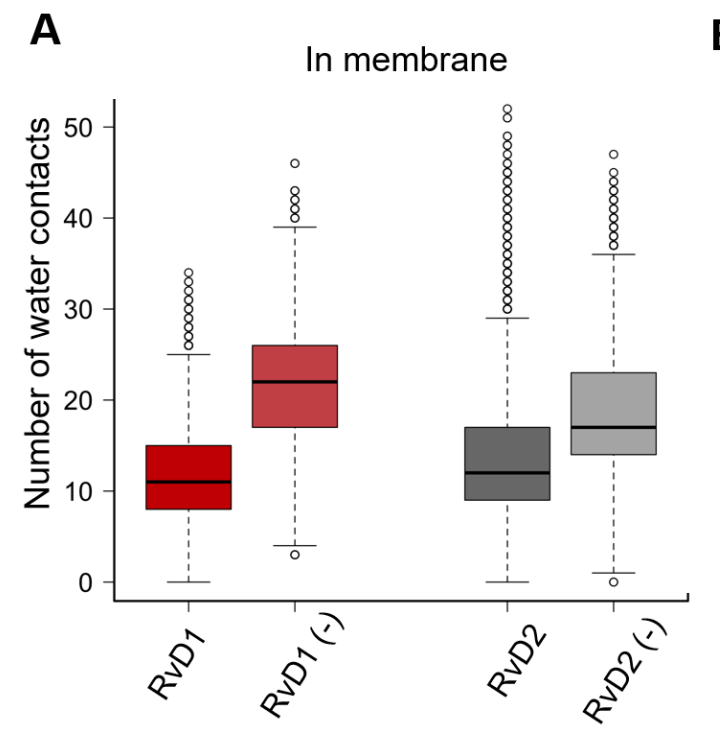

B In bulk water

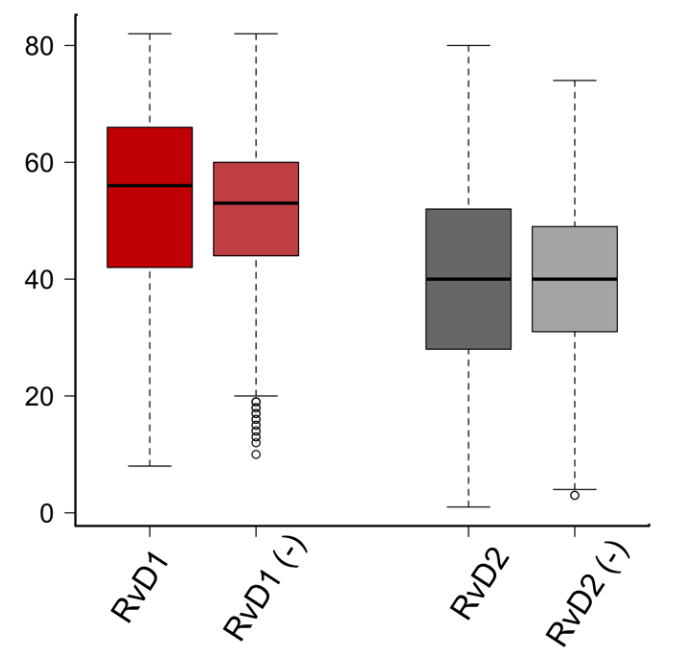

Fig. S4. The number of water molecules within $4 \AA$ of the neutral and charged species (denoted by a negative sign) of RvD1 and RvD2 in their preferred bilayer locations as well as in the bulk aqueous phase. A) The number of water contacts is significantly higher for the charged species than the neutral species when they are solvated in the membrane. B) However, in the bulk water, the contacts are much higher for RvD1 than RvD2 irrespective of their ionization states. 